wegungen der Digitalisierung, den Zustand von Gesellschaft (und nicht nur der US-amerikanischen) machen, lohnt sich diese Mühe der Rekonstruktionsarbeit.

\section{Gernot Wersig}

\section{Wiebke Müller \\ Journalistenausbildung in Europa}

Bestandsaufnahme, neue Modelle, Entwicklungsperspektiven

Berlin: Vistas, 1999. - 223 S.

(Reihe Vistascript; 13)

ISBN 3-89158-231-5

"Journalistenausbildung in Europa“ - unter diesem Titel erwartet der Leser zunächst einen Überblick über die (durchaus heterogenen) Strukturen des Berufsfeldes in verschiedenen europäischen Ländern und die jeweils dazugehörenden Aus- und Weiterbildungssysteme. Darüber erfährt man in dem lesenswerten Buch von Wiebke Müller relativ wenig. Denn die Autorin will mehr: Sie interessiert sich für Ausund Weiterbildungsangebote, die einen inhaltlichen oder strukturellen Europa-Bezug aufweisen, also entweder europäische Themen in ihr Curriculum integrieren oder gar europäische Ableger oder transnationale Kooperationen entwickeln.

Die Beschäftigung mit Aspekten der europäischen Integration hat häufig mit einem besonderen normativen Überschuss zu kämpfen: „Europa“ ist irgendwie immer „gut“, „mehr Europa“ (im Sinne von mehr Kooperation und Integration) deshalb scheinbar automatisch besser. Die Gefahr einer Idealisierung von Initiativen und Modellen, die "mehr Europa“ wollen, liegt deshalb nahe. Wiebke Müller begegnet dieser Gefahr auf einleuchtende Weise. Sie beschreibt natürlich mit großer Sympathie die bestehenden Angebote in diesem Bereich und die sich entwickelnden transnationalen Netzwerke. Aber sie fragt zugleich nach verschiedenen Stolpersteinen: nach den Erfahrungen, die die Ausbildungsinstitutionen mit Europa als Thema und Kooperationsfeld tatsächlich gemacht haben (S. 107 ff.), nach den im Moment durchaus noch schmalen Beschäftigungsfeldern für Journalistinnen und Journalisten mit Europa-Qualifikation (S. 173 - 186), nach der Abhängigkeit europäischer Vernet- zungsinitiativen von den nicht immer gleich stark sprudelnden EU-Förderquellen usw.

Das wichtigste länderübergreifende Netzwerk der Journalistenaus- und -weiterbildung stellt die 1990 gegründete „European Journalism Training Association" (EJTA) dar, die ihren Sitz soeben von Maastricht nach Bordeaux verlegt hat Im Rahmen der EJTA-Kooperationen haben sich auch die beiden bekanntesten transnationalen Bildungsangebote für (angehende) Journalisten entwickelt: die „Euroreporter-Seminare, bei denen Journalistenschüler aus verschiedenen europäischen Ländern gemeinsam während einer Woche ein journalistisches Produkt (z. B. eine Zeitschrift oder ein TV-Magazin) zu einem europäischen Rahmenthema erstellen, sowie die „Europafilialen“ des „Centre de Formation des Journalistes (CFJ) in Paris, die es Absolventen im Fach Journalismus (nicht nur) aus Europa ermöglichen, sich in einem Jahr als „Europajournalisten" zu spezialisieren.

Die Seminare sind für die Autorin das Paradebeispiel für einen inhaltlichen Europa-Bezug in der Journalistenausbildung, während die Europafilialen den Prototyp einer Verbindung von inhaltlichem und strukturellem EuropaBezug darstellen, der inzwischen verschiedene Nachahmer gefunden hat.

Die Bedeutung der EJTA als Rahmen für diese Aktivitäten hat auch dazu geführt, dass die Autorin die Mitglieder dieses Netzwerkes als wichtigsten empirischen Einstiegspunkt für ihre empirische Untersuchung gewählt hat. Den Kern ihrer Arbeit bilden drei einander ergänzende schriftliche Befragungen: Angeschrieben wurden erstens die Mitgliedsinstitutionen der EJTA, zum Zweiten die deutschen Ausbildungseinrichtungen für Journalisten, die nicht Mitglied der EJTA sind, und drittens für den Bereich der PR-Ausbildung die Mitglieder der Confédération Européenne des Relations Publiques (CERP). Die Rücklaufquoten lagen zwischen 40 und 50 Prozent und sind damit für diese Art der (europaweiten!) Befragung durchaus annehmbar. Allerdings ist die absolute Zahl der eingegangenen Fragebögen (32 EJTA-Mitglieder, 13 EJTA-Nicht-Mitglieder in Deutschland und 21 CERP-Ausbildungseinrichtungen) so gering, dass sich bei manchen Fragen Fallzahlen ergeben, die nicht hätten interpretiert werden sollen. Auch die Darstellung von Prozentverteilungen ist bei Fallzahlen unter 100 nicht sonderlich sinnvoll. Doch die Au- 
torin stützt sich nicht auf diese Befragungsdaten allein: Ergänzend hat sie Dokumentenauswertungen und Gesprächsrecherchen durchgeführt und Stellenausschreibungen im "Journalist" im Hinblick auf die im Berufsfeld geforderten Europa-Qualifikationen vorgenommen.

Interessant und auch ein wenig ernüchternd ist das Ergebnis, dass die Ausbildungsinstitutionen die Prioritäten für die Zukunft vor allem im Bereich solider Fachkompetenz (Handwerk, Technik) und Berufsethik sowie bei den Fremdsprachenkenntnissen sehen, weniger dagegen bei der Vermittlung interkultureller Kompetenzen oder der Schaffung gemeinsamer europäischer Ausbildungsstandards (S. 109). Dieses Ergebnis trifft in der Tendenz auch für die befragten PR-Ausbildungseinrichtungen zu und ist ein Indiz dafür, dass die "hehren“ Ziele einer weiter gehenden europäischen Integration in der Praxis der Ausbildung für Kommunikationsberufe von eher untergeordneter Bedeutung sind. Auch europabezogene Kompetenzen werden zumindest im Journalismus noch keineswegs häufig verlangt. Nur in $10 \%$ der untersuchten Stellenanzeigen für Journalisten wurden solche Kompetenzen gefordert, während ein Europa-Profil immerhin in rund $30 \%$ der PR-Stellenanzeigen erwartet wird
(S. 184). Bemerkenswert ist schließlich folgendes Fazit: Zwar haben erwartungsgemäß internationale Netzwerke wie EJTA oder CERP eine Katalysatorwirkung für die Erarbeitung und Durchführung europaorientierter Ausund Fortbildungskonzepte. Ausbildungseinrichtungen, die innerhalb solcher Netzwerke bereits Erfahrungen mit europäischer Kooperation und europäischen Inhalten gemacht haben, schätzen die Arbeitsmarktchancen von Journalisten und Kommunikationsberuflern mit internationalem Ausbildungsprofil allerdings tendenziell kritischer ein als Einrichtungen, die nicht Mitglied in einem solchen Netzwerk sind.

Die Arbeit von Wiebke Müller enthält zum einen viele Informationen, die sonst aufwändig zusammengesucht werden müssten. Sie kann durch den Adressenteil am Schluss und die Beschreibung einzelner Europa-Programme zum Teil auch als Nachschlagewerk dienen. Die Arbeit ernüchtert und öffnet am Ende doch den Blick für mögliche zukünftige Betätigungsfelder. Dazu zählt die Autorin unter anderem die Analyse der Ausbildungssituation in Mittel- und Osteuropa sowie Modelle der Weiterbildung für Journalisten und Kommunikationsberufler in Europa.

Hartmut Weßler 